\title{
Los avances de la jurisprudencia internacional contra la impunidad de las violaciones en los conflictos armados
}

\author{
The advances of the international \\ jurisprudence against the impunity of the \\ violations in the armed conflicts
}

Soledad Torrecuadrada García-Lozano*

\begin{abstract}
RESUMEN
Estudiar los crímenes de los que específicamente son víctimas las mujeres en el Derecho Penal Internacional es una tarea muy dura, debido a su crueldad y a la tolerancia con que se contemplan. En los últimos tiempos, los crímenes de naturaleza sexual son un instrumento de guerra muy barato y de gran eficacia. A pesar de ello, en los últimos años del siglo xx, la movilización social, debido al conocimiento casi inmediato de las brutalidades cometidas en los conflictos armados, ha provocado la sensibilización hacia estos comportamientos y el rechazo de su impunidad. Avanzamos en su represión, aunque no ocurre lo mismo con la prevención ni con la reparación a las víctimas, ámbitos materiales que aún son asignaturas pendientes.
\end{abstract}

Palabras clave: Violación - Crimen de guerra - lesa humanidad - genocidio - consentimiento - Derecho Penal Internacional

Soledad Torrecuadrada García-Lozano es Profesora titular de Derecho Internacional y Relaciones Internacionales en la Universidad Autónoma de Madrid (2000).

S.torrecuadrada@uam.es. Recibido: 2I de abril de 20I8. Aceptado: I5 de mayo de 2018. 


\section{ABSTRACT}

Studying the crimes of which women are specifically victims in International Criminal Law is a very difficult task, due to the cruelty and the tolerance with which they are contemplated. In recent times, crimes of a sexual nature are a very cheap and highly effective instrument of war. In spite of this, the social mobilization at the end of the $20^{\text {th }}$ century, due to the near immediate knowledge of the brutalities committed in armed conflict has given rise to more awareness of these behaviours and the rejection of impunity in relation to these crimes. While we are making advances with respect to their repression, the same thing cannot be said about their prevention and reparation, issues that are still unresolved matters.

Keywords: Rape - War crimes - Crimes against humanity Genocide - Consent - International Criminal Law. 
Soledad Torrecuadrada García-Lozano

Los avances de la jurisprudencia internacional contra la impunidad de las violaciones en los conflictos armados

\section{INTRODUCCIÓN}

Los conflictos armados provocan importantes daños físicos, psicológicos o ambos entre quienes logran sobrevivir a ellos, especialmente en los más recientes, caracterizados "por unos niveles estremecedores de brutalidad y un burdo desprecio por la vida y la dignidad humana" (Párrafo 4 del undécimo Informe del Secretario General de Naciones Unidas sobre la protección de los civiles en los conflictos armados, Doc. $S / 2015 / 453$, del I8 de junio de 20I5), evidenciada en las víctimas de los crímenes sexuales. Tradicionalmente, las violaciones eran un daño colateral del conflicto armado, formando parte del botín de guerra (el informe en: http://www.un.org/womenwatch/ daw/public/w2apr98.htm\#part2), justificadas en el impulso natural de los combatientes. En la última década del siglo xx, estos crímenes han dejado de ser invisibles e impunes y hemos adquirido conciencia de su existencia y de la necesidad de la adopción de medidas para su prevención y represión.

$\mathrm{Ni}$ la violencia sexual en los conflictos armados ni los propósitos perseguidos con ella son una novedad (Párrafo 7 del Informe Preliminar sobre las formas contemporáneas de la esclavitud en doc. E/CN.4/Sub.2/1996/26, del I6 de julio de 1996), a diferencia de lo que ocurre con la búsqueda del fin de la impunidad de estos crímenes, que sí lo es. Solo al final del siglo pasado, la coincidencia de múltiples elementos provocó la adopción de medidas que supusieran el inicio del camino para prevenir y reprimir estos crímenes. Los tribunales penales internacionales (TPIS) han sido un eslabón relevante, aplicando el Derecho Penal Internacional (DPI), interpretando conceptos jurídicamente indeterminados cuando ha sido preciso, con el propósito de determinar la responsabilidad penal de los autores de estos crímenes cuando han tenido ocasión de conocer de ellos. Entre los textos constitutivos de estos tribunales merece destacarse el Estatuto de Roma, por ser el primero de naturaleza convencional en tipificar los crímenes sexuales como una manifestación de la lesa humanidad ${ }^{1}$ y del crimen de guerra, siempre que se cometa "como parte de un ataque generalizado o sistemático contra la población civil o contra una parte de ella» (respectivamente, artículos 7.I.g) y 8.2.b) apartado xxii y d) apartado vi.). No se incorpora expresamente como genocidio, pero puede desprenderse de la interpretación del artículo sexto de este texto. Sin embargo, el Fiscal de la Corte Penal Internacional (CPI) ha defraudado en este punto, pues en el

1 Antes lo fue por el Council Control Law № ro, Punishment of persons guilty of war crimes, crimes against peace and against humanity, cuyo artículo 2.I.c reproducía la definición de lesa humanidad de los estatutos de los tribunales de Nuremberg y Tokio, incorporando la violación como uno de los comportamientos constitutivos del tipo. En http://avalon.law.yale.edu/imt/ imtro.asp. 
asunto Lubanga excluyó los crímenes de naturaleza sexual de la acusación, a pesar de que la mayoría de los niños soldados habían sido víctimas de esclavitud sexual, impidiendo con ello avanzar en la persecución de la responsabilidad de los autores de estos comportamientos (Bou, 20I2 y Días, 20I4).

En todo caso, la tipificación de estos crímenes es un hito en el camino que persigue la erradicación de la violación y las agresiones sexuales como un instrumento de guerra muy barato $\mathrm{y}$ eficaz ${ }^{2}$; así como la reinserción de las víctimas en sus comunidades de origen, ambos al día de hoy están lejos de alcanzarse. En este sentido, la concepción tradicional de la violación se entiende como un comportamiento que atenta contra el honor (Convenios de Ginebra), pero alcanza no solo al honor personal (bien jurídico individual), sino también al familiar o incluso al de la propia comunidad (bien jurídico colectivo). En algunas culturas, de esta dualidad conceptual derivan problemas para la víctima, a quien se culpabiliza del daño sufrido en el bien jurídico colectivo: la deshon-

2 Informe de Zainab Hawa Bangura, "La violencia sexual: un instrumento de guerra”, en http://www.rescue.org/blog/confronting-rape-a-weapon-war. También el Consejo de Seguridad ha reconocido la utilización de la violencia sexual como arma de guerra en su resolución I820 (2008). Asimismo, Informe de Amnistía Internacional titulado Vidas rotas. Crímenes contra mujeres en situación de conflicto, de 2004 , página I7, en: https://www.amnesty.org/es/ documents/ACT77/075/2004/es/ ra de toda la familia o la comunidad (Kucukalic, 20I4), priorizando así a las víctimas colectivas sobre las individuales (OMAnYondo y otros, 2005), olvidadas o socialmente excluidas, que sobreviven al desprecio de familiares y vecinos, frente a otros mutilados o heridos, considerados héroes.

Desafortunadamente, carecemos de datos concretos sobre las agresiones sexuales que se producen en el transcurso de los conflictos aunque, según el Secretario General, la "violencia sexual, por razón de género, está muy generalizada". Las cifras son aproximadas, porque muchas de las víctimas eligen no denunciar por miedo a la estigmatización que ello puede producir, prefiriendo vivir en silencio con las consecuencias de este delito a las que derivarían de su exposición.

Si nos retrotraemos a la Segunda Guerra Mundial, se han documentado violaciones de mujeres rusas por parte de alemanes y de alemanas por los aliados cuando tomaron Berlín ${ }^{3}$. Más recientemente, en el conflicto que concluyó con la independencia de Bangladesh, se estima que hubo entre doscientas y cuatrocientas mil mujeres violadas (AKman, 20I3 y Frieyro de

3 http://www.elmundo.es/cronica/2015/03/08/ 54fadb85268e3ee05I8b4570.html o http:// www.abc.es/cultura/20150302/abci-segund a - g u e r r a - m u nd i a l-viol a c i o nes-201503021049.html. Sobre la situación de las mujeres en la Alemania de la posguerra mundial puede verse Anónimo (2005), Una mujer en Berlín, Anagrama, Barcelona. 
Soledad Torrecuadrada García-Lozano

Los avances de la jurisprudencia internacional contra la impunidad de las violaciones en los conflictos armados

LARA, 20I2), también en Sri Lanka ${ }^{4}$ o en Timor (ChInkin, 1994), entre otros. En los conflictos en el territorio de la antigua Yugoslavia y en Ruanda, se produjeron embarazos forzados, o las violaciones por estadounidenses en Iraq ${ }^{5}$. Esos crímenes sexuales no siempre son atribuibles a las tropas enemigas, también a las amigas, como se puso de relieve en la guerra en Katanga, en la que los miembros de la UNTAC ${ }^{6}$ cometieron estos delitos o las tropas de Nu en Somalia (Ndulo, 2009). Comportamiento, este último, mucho más criticable, si cabe, al vulnerar también la obligación de protección a la población civil en la que encuentra fundamento su despliegue, por lo que las sanciones en estos casos deberían ser especialmente duras y ejemplarizantes.

4 Informe de Amnistía Internacional respecto de los crímenes sexuales cometidos en este conflicto: http://www.amnesty.org/ en/library/asset/ASA37/008/1996/en/ c8Ib26f3-ebor-IIdd-aadi-ed57e7e5470b/ asa370081996en.html

5 http://argentinatoday.org/2016/or/22/violacion-de-mujeres-iraquies-por-las-fuerzas-de- estados-unidos-como-arma-de-guerra/; https://yahel.wordpress. com/2013/rI/3o/irakviolacion-de-las-mujeres-iraquies-por-parte-de-las-fuerzas-de-losestados-unidos-como-arma-de-guerra-fotos/; incluso en tiempo de paz en las bases estadounidenses: http://www.revcom. us/a/305/sobre-la-epidemia-de-violaciones-en-las-fuerzas-armadas-estadounidenses-es.html

6 Informe redactado en 1962 por Ernest Van den Haag, The War in Katanga, Report of a Mission, p. Io. https://archive.org/stream/ TheWarInKatangaTheUnitedNationsInTheCongoReportOfAMission/Katangaz\#page/niI/mode/2up/search/rape
Es evidente que los abusos sexuales y la violación vulneran el Derecho Humanitario, el DPI y los derechos inherentes a la dignidad humana, pudiendo el mismo comportamiento responder a un tipo distinto en función del contexto o la intención perseguida con él: crimen de lesa humanidad, un crimen de guerra o incluso genocidio. Cuando nos encontramos con etnias patrilineales, al seguir el hijo la etnia del padre, el embarazo forzoso se emplea como un instrumento que contribuye a la desaparición del grupo materno (a diferencia de otros grupos matrilineales, como los bibri (en Costa Rica), los garo (en el noroeste de la India), los mosuo (en China) los manan kaban (en Sumatra) o los akran (en Ghana). Rodríguez, I99I), resultando constitutivo de genocidio. En este sentido se pronunció el Tribunal Penal Internacional para Ruanda (en adelante TPIR) en el caso Akayesu?

En las páginas siguientes analizaremos la actualidad jurisprudencial, pues si bien se ha reconocido que los crímenes sexuales vulneran una norma imperativa, sus autores solo responderán penalmente si se han realizado con la finalidad de cometer genocidio, crímenes de lesa humanidad o crímenes de guerra, para terminar, como es habitual en estos trabajos, con un apartado dedicado a conclusiones.

7 Sentencia Akayesu (Prosecutor v. Jean Paul Akayesu, case n. ICTR-96-4-T, de 2 de septiembre, parágrafos 494 y 73I, especialmente. 


\section{La Criminalización de la violación en el Derecho Penal INTERNACIONAL}

Sorprende la ausencia de criminalización de los crímenes sexuales en el DPI hasta su incorporación a la competencia de los tribunales creados $a d$ hoc por el Consejo de Seguridad. Entonces, la confluencia de múltiples factores provocó una vertiginosa evolución en este sentido (Ambos, 20I2), entre los que merecen destacarse la conciencia de los brutales comportamientos, así como el cambio de paradigma que ha transformado los crímenes contra el honor en crímenes contra la dignidad humana.

El funcionamiento de estos tribunales inició la exigencia de responsabilidad penal de los autores de estos crímenes, y su jurisprudencia ha colmado las lagunas que existían, hasta entonces, en algunos conceptos relevantes, incluso en su tipificación. Con ello se ha ubicado a la víctima del crimen en el centro de la escena, identificando la autonomía sexual como elemento relevante en lugar del honor individual y familia. Para ello, los TPIs, principalmente los tribunales de Yugoslavia (TPIY) y de Ruanda (TPIR), han superado las indefiniciones conceptuales y las barreras que inicialmente dificultaban el procesamiento de los autores de estos crímenes.

La jurisprudencia ha provocado un progreso exponencial en la criminalización de los crímenes sexuales, lo que ha de valorarse positivamente, al considerar la violación una vulneración de las normas de ius cogens, lo que no ha repercutido — desafortunadamente- - sobre la reparación a las víctimas que continúan siendo estigmatizadas, marginadas, invisibles cuando no repudiadas, en su comunidad ${ }^{8}$. Debemos atender a estas mujeres adecuadamente, como requiere su estatuto de víctima, debiendo acompañarse la exigencia de responsabilidad penal del autor del comportamiento de la protección de las víctimas reales o potenciales, lo que implica la aplicación de mecanismos al efecto, tanto durante los combates como después de ellos.

Por otra parte, estos crímenes sexuales son una manifestación de genocidio, de crímenes contra la humanidad o de guerra o de tortura, si las circunstancias que rodean su elemento material (la violación) permiten esta consideración, resultando determinante a estos efectos el elemento subjetivo o contextual para estos propósitos. Pese a la acción de los TPIs, los crímenes sexuales no han dejado de utilizarse como arma de guerra muy eficaz y barata. Los informes que remite el Secretario General al Consejo

8 Por ejemplo, respecto del olvido de las víctimas, Esma Kucukalic Ibrahimovic "Las mujeres violadas en la Guerra de Bosnia, dobles víctimas del conflicto veinte años después", en Instituto de Estudios Estratégicos, 35/20I4, de 7 de abril, en http://www. ieee.es/Galerias/fichero/docs_opinion/20I4/DIEEEO35-20I4_GuerraBosnia_DDHH_EsmaKucukalic.pdf 
Soledad Torrecuadrada García-Lozano

Los avances de la jurisprudencia internacional contra la impunidad de las violaciones en los conflictos armados

de Seguridad sobre la violencia sexual relacionada con los conflictos ${ }^{9}$, avergüenza por los datos que aporta, tanto desde una perspectiva cuantitativa como cualitativa, por la barbarie de la que se hace gala y la cada vez más corta edad de las víctimas ${ }^{10}$.

Observemos que el miedo a las violaciones puede provocar desplazamientos poblacionales, máxime cuando esas agresiones se producen de forma pública, o al menos delante de los familiares de las víctimas (DE VITO y otros, 2009). En ocasiones, estos comportamientos son más eficaces para derrotar psicológicamente al enemigo que el logro de objetivos militares, debido a la afectación de un bien jurídico individual (la víctima) y colectivo (la comunidad a la que pertenece) en las culturas mencionadas supra.

Los Estatutos de los TPIs confieren competencia a estos órganos para co-

9 Vid. El último de ellos ( $\mathrm{S} / 2015 / 203)$ en http:// www.un.org/es/comun/docs/?symbol $=\mathrm{S} / 2015 / 203$

10 Véase por ejemplo las sentencias del Tribunal de Yugoslavia en el caso de la masacre de Foça, del I2 de junio de 2002 en los asuntos IT-96-23 e IT-96-23/I-A, Fiscal c. Dragoljub Kunarac, Radomir Kovac y Zoran Vukovic de la Sala de Apelaciones en http://www.icty.org/x/cases/kunarac/ acjug/en/kun-ajo206r2e.pdf o la sentencia del Tribunal Penal para Ruanda, en el caso del Fiscal c. Jean-Paul Akayesu, de la Sala Primera en http://unictr.unmict.org/sites/ unictr.org/files/case-documents/ictr-96-4/ trial-judgements/en/980902.pdf nocer de las violaciones producidas debido a la crueldad de lo ocurrido en sus respectivos territorios, y su creación, ex post facto, incidió sobre el alcance de sus competencias. Así, los Estatutos del tpiy (artículo 5.g) y del TPIR (artículo 3.g), como el Estatuto de Roma, reconocen la violación como crimen de lesa humanidad ${ }^{11}$. De los primeros se desprende su consideración como crimen de guerra (artículo 2.c TPIY y 4.a TPIR), así como expresamente en el Estatuto de Roma [ver artículo 8.2.b) xxii) y 8.5.e) vi)]. Por lo demás, el Estatuto del tPIY es más restrictivo que el TPIR en lo que a su consideración como crimen de lesa humanidad se refiere, exigiendo aquel la existencia de un nexo entre el crimen y el conflicto en el transcurso del cual se comete.

11 Cuyo artículo 7.I) g) establece que una de las conductas consideradas como tal es la "violación, esclavitud sexual, prostitución forzada, embarazo forzado, esterilización forzada o cualquier otra forma de violencia sexual de gravedad comparable". 


\section{LA VIOLACIÓN COMO CRIMEN DE GENOCIDIO}

La Corte Internacional de Justicia (CIJ) en el asunto sobre la Convención contra el Genocidio (sentencia del 26 de febrero de 2007), advirtió el acuerdo de las partes en conflicto acerca de que las violaciones y las agresiones sexuales pueden ser constitutivas de genocidio siempre que tengan carácter sistemático (párrafo I65 de la Sentencia de la CIJ del 3 de febrero de 2015 , en el asunto de la aplicación de la convención para la prevención y represión del crimen de genocidio - Croacia c. Serbia-) y vayan "accompanied by a specific intent to destroy the protected group" (párrafo 300), lo que resulta aplicable no solo al caso yugoslavo, sino también al ruandés, de conformidad con la jurisprudencia de ambos tribunales.

El TPIR, en su Sentencia en el caso contra Jean-Paul Akayesu (párrafo 73I) no se limitó a reconocer que las violaciones pueden ser constitutivas de genocidio "as long as they were committed with the specific intent to destroy, in whole or in part, a particular group, targeted as such", sino también abordó la aplicación de los elementos constitutivos del crimen de genocidio a los crímenes sexuales: subjetivo (intencionalidad) y material (violación). La intencionalidad de "destruir, total o parcialmente, a un grupo nacional, étnico, racial o religioso" (artículo 2 de la Convención sobre Genocidio), es fundamental para calificar el comportamiento como genocidio. Para el Estatuto de
Roma (artículo 30) es imprescindible la presencia de la finalidad de producir las consecuencias (desaparición total o parcial del grupo) que de ella derivan. El propósito destructor de un grupo humano es el elemento distintivo respecto del asesinato, y no siempre resulta evidente, salvo que contemos con documentación que lo demuestre o que la intención genocida pueda deducirse de otros elementos, como:

"From the general context of the perpetration of other culpable acts systematically directed against that same group, whether these acts were committed by the same offender or by others. Other factors, such as the scale of atrocities committed, their general nature, in a region or a country, or furthermore, the fact of deliberately and systematically targeting victims on account of their membership of a particular group, while excluding the members of other groups, can enable the Chamber to infer the genocidal intent of a particular act" (párrafo 523).

La intencionalidad genocida debe ser previa tanto a la violación como a la adopción de medidas para impedir nacimientos dentro de un grupo, según la Sala II del Tribunal de Ruanda en la sentencia contra Kayisema y Ruzinda- 
Soledad Torrecuadrada García-Lozano

Los avances de la jurisprudencia internacional contra la impunidad de las violaciones en los conflictos armados

$\mathrm{na}^{12}$. Así, la destrucción del grupo puede conseguirse mediante procedimientos físicos que conduzcan a la esterilización, pero también como consecuencia de la transmisión de enfermedades que provoquen este resultado o con medios psicológicos, entendiendo así la capacidad de convencer a las mujeres para que rechacen tener descendencia al regreso a su comunidad ${ }^{13}$.

El elemento material del genocidio se produce con las violaciones masivas y sistemáticas, que en el caso ruandés tuvo como víctimas a mujeres tutsis, ya que perseguían la eliminación de este grupo humano. Quedarían excluidas del tipo, las violaciones de mujeres de cualquier otra etnia, por carecer del animus de destrucción del grupo, al ser esta intención precisamente la que define el crimen de genocidio (su elemento contextual). La Sala Primera del TPIR no niega la existencia del delito de violación, pero queda al margen del tipo

12 Párrafo 9r de la Sentencia de la Sala II del TPIR, en el asunto Fiscal c. Clement Kayishema y Obed Ruzindana, del 2I de mayo de 1999, Caso no ICTR -95-I-T, en http:// unictr.unmict.org/sites/unictr.org/files/ case-documents/ictr-95-I/trial-judgements/ en/99052I.pdf

13 Id. Nota anterior en parráfo 508, en el inmediatamente precedente (507), el Tribunal indica además que: "In patriarchal societies, where membership of a group is determined by the identity of the father, an example of a measure intended to prevent births within a group is the case where, during rape, a woman of the said group is deliberately impregnated by a man of another group, with the intent to have her give birth to a child who will consequently not belong to its mother's group". en el que se subsumió en este momento ese comportamiento (genocidio).

Las aportaciones del TPIR han sido muy relevantes en relación con los elementos del crimen de genocidio, que hasta entonces permanecían sin una clara concreción, incluso se han incorporado en la jurisprudencia del TPIY y de la CIJ, evidenciando así una coordinación insospechada ante la tan temida fragmentación, anunciada consecuencia de la proliferación de TPIs. A pesar de esto, han existido discrepancias entre ellos, como la relativa al concepto de violación, donde los TPIY y TPIR, utilizaron contenidos conceptuales diferentes: el primero la definió atendiendo a los comportamientos constitutivos del delito, mientras el segundo prefirió dotarla de un alcance más amplio. Esto llevó a ambos tribunales a entablar una discusión a través de su jurisprudencia sobre el concepto utilizado por el otro ${ }^{14}$.

14 El tpiy, en el párrafo I60 de la Sentencia del ro de diciembre de I998, dictada en el asunto contra Anto Furundzija (IT-95I7/I-T), respondió de este modo el concepto indicado por el TPIR en el caso Akayesu: "This definition was regarded by Trial Chamber I of ICTR, in Prosecutor v. Jean Paul Akayesu, hereafter "Akayesu", as sic et simpliciter applying to any rule of international law on torture, including the relevant provisions of the ICTR Statute"; en la Sentencia de 27 de enero de 2000 , de este mismo tribunal, en el asunto Alfred Musema ( ${ }^{\circ}$ ICTR96-I3-T) afirma suscribir "à l'approche conceptuelle de la définition du viol retenue dans le Jugement Akayesu, qui reconnait que l'essence du viol ne réside pas dans le détail des parties $d u$ corps et des objets qui interviennent dans sa commission, mais plutôt dans le fait qu'il 
Hemos de calificar positivamente la consideración de las violaciones como actos de genocidio, aunque nos plantee cuestiones como su propia conceptualización. El genocidio es un crimen colectivo, contra un grupo humano, mientras la violación es necesariamente un acto individual, de los miembros del grupo al que se pretende aniquilar, tan individual como otros igualmente considerados como actos de genocidio, por ejemplo los traslados de niños a otro grupo o las medidas para impe-

constitue une agression à caractère sexuel commise sous l'empire de la contrainte". Es el párrafo 226, por su parte, el mismo TPIY, en la Sentencia de primera instancia contra Dragoljub Kunarac, de 22 de febrero de 200I, (IT-96-23-T \& IT-96-23-I-T) entiende (en el parágrafo 460) demasiado estricto el concepto avanzado en Furundzija al establecer que solo existe violación cuando va acompañada de uso de la fuerza o de su amenaza, en este caso, el hecho físico de la violación "a lieu sans le consentement de la victime. Le consentement à cet effet doit être donné volontairement et doit résulter de l'exercice du libre arbitre de la victime, évalué au $v u$ des circonstances. L'élément moral est constitué par l'intention de procéder à cette pénétration sexuelle, et par le fait de savoir qu'elle se produit sans le consentement de la victime”. La Sentencia de la Sala de primera instancia III, del 15 de mayo de 2005 del TPIR contra Laurent Semanza (ICTR-9720-T), párrafo 345, se muestra convencida por la conclusión alcanzada en el caso Kunarac, aplicándola al caso concreto : «Ce faisant, la Chambre reconnaît que, sans satisfaire cette définitions étroite, d'autres actes de violence sexuelle (torture, persécution, réduction en esclavage ou autres actes inhumains) peuvent faire l'objet de poursuites en tant qu'autres crimes contre l'humanité ressortissant la compétence du Tribunal de crans". Sobre esta discrepancia doctrinal puede verse Bou (20I2). dir nacimientos dentro de aquel. De la consideración de estos supuestos como genocidio (violación genocida en palabras de Clare McGlyn, 2008, 79) se desprende la relevancia del grupo más que la de cada una de las víctimas. De todos modos, la apreciación puede tener un efecto positivo sobre estas últimas, pues de su conceptualización deriva la idea de haber sido violadas no en tanto que mujeres, sino como unidades dentro del grupo étnico perseguido. Psicológicamente, la repercusión puede ser diferente, aunque la comunidad a la que pertenecen no comparta del todo esta visión y continúen estigmatizándolas, como ha ocurrido tanto en Ruanda como en la antigua Yugoslavia, por solo citar dos ejemplos. 
Soledad Torrecuadrada García-Lozano

Los avances de la jurisprudencia internacional contra la impunidad de las violaciones en los conflictos armados

\section{LA VIOLACIÓN COMO TORTURA}

La jurisprudencia de los TPIs también ha considerado la violación como una forma de tortura. Es el caso de la Sentencia Akayesu, en la que se considera que la violación y las agresiones sexuales encajan en la definición que del tipo contiene el artículo i de la Convención Contra la Tortura y Otros Tratos o Penas Crueles, Inhumanos o Degradantes, pues se provocan "dolores o sufrimientos graves" físicos o psicológicos, con el propósito de "intimidar o coaccionar a esa persona o a otras", por el solo hecho de pertenecer a un grupo humano determinado. En consecuencia, el motivo del comportamiento es la discriminación.

En cuanto al elemento objetivo del tipo, el TPIY consideró la violencia sexual como un atentado grave a la integridad física o mental de las víctimas, al analizar el alcance del artículo 4.2.b) de su Estatuto, afirmando que del mismo modo que ocurre con los actos constitutivos de:

\section{"torture, de traitements inhumains ou} dégradants, de violences sexuelles, $y$ compris les viols, la santé de la victime ou se traduisant par une défiguration ou des blessures" (Sentencia de la Sala de Primera Instancia de 3I de julio de 2003 en el caso Stakic (Iт-97-24), párrafo 516).

Los crímenes sexuales reúnen los elementos de intencionalidad, gravedad del sufrimiento de la víctima y la finalidad ${ }^{15}$. El TPIR considera que, aunque la Convención contra la tortura no incorpore la violación en la definición de tortura de forma específica, no debe descartarse por su propósito de:

"intimidation, degradation, bumiliation, discrimination, punishment, control or destruction of a person. Like torture, rape is a violation of personal dignity, and rape in fact constitutes torture when inflicted by or at the instigation of or with the consent or acquiescence of a public official or other person acting in an official capacity" (párrafo 597 de la Sentencia Akayesu).

En consecuencia, igual que en el genocidio, solo las violaciones que reúnen los elementos constitutivos de la tortura podrán considerarse a estos efectos. Teniendo en cuenta lo anterior, también el TPIY (Sala de Apelaciones) consideró culpables de tortura a los acusados debido a:

"souffrance physique, la peur, l'angoisse, lincertitude et l'humiliation auxquelles les Appelants ont à plusieurs reprises soumis leurs victimes,

15 La síntesis de elementos del concepto de tortura se encuentran en el apartado $182 \mathrm{de}$ la Sentencia de I2 de junio de 2002, de la Sala de la Sala de apelaciones del Tribunal de Yugoslavia, en el caso Fiscal contra Dragoljub Kunarac, Radomir Kovac y Zoran Vukovic, IT-96-23 \& IT-96-23/I-A. 
font de leurs actes des actes de torture. Il ne s'agissait pas de cas isolés" (parágrafo I85 de la sentencia del TPIY contra Kunarac, Kovac y Vukovic).
Por último, en tanto las violaciones y los abusos sexuales son tratos inhumanos o degradantes, en un contexto de conflicto armado cabe exigir la responsabilidad penal de sus autores tanto por los tribunales nacionales como subsidiariamente por la CPI.

\section{LA VIOLACIÓN COMO CRIMEN DE LESA HUMANIDAD}

Los TPIs han considerado y subrayado la violación y las agresiones sexuales como una materialización del crimen de lesa humanidad. Tanto los Estatutos de los tribunales ad hoc (artículo 5 en el caso de Yugoslavia o el artículo 3 de Ruanda, este último añade al anterior: "por razones de nacionalidad o por razones políticas, étnicas, raciales o religiosa") como el Estatuto de Roma (artículo 7. I. g) así lo establece, siempre que sea parte de un "ataque generalizado o sistemático contra una población civil y con conocimiento" de formar parte de esta empresa de quien lo comete. La jurisprudencia de estos tribunales ha profundizado en esta caracterización y en los elementos precisos para la consideración de los comportamientos que nos ocupan como crimen de lesa humanidad.

En este punto, la diferencia contextual existente entre el crimen de genocidio y la lesa humanidad se encuentra en que en este último ha de tratarse de un ataque generalizado y masivo contra la población civil con independencia de cuál sea su nacionalidad o el grupo étnico al que pertenezca, pues lo relevante es que esa población resulte ser víctima principal del ataque y no de forma incidental ${ }^{16}$. Ha de dirigirse contra un número elevado de víctimas, colectivo y de una gravedad considerable $^{17}$ para ser un comportamiento constitutivo de lesa humanidad.

Debido a que nos encontramos ante un tipo delictivo colectivo, los actos individuales que conforman el ataque generalizado o sistemático, deben apreciarse dentro de su respectivo contex-

16 Esta es una afirmación reiterada por la CPI en su jurisprudencia, entre ella, en el párrafo 399 de la decisión de la Sala Preliminar I en el caso contra Germain Katanga (ICC-0I/04-0I/07-717-T), o en el párrafo 76 de la Decisión de la Sala Preliminar II, en el asunto contra Jean-Pierre Bemba Gombo (ICC-0I/05-0I/08) pero que no es novedosa pues el TPIY ya la había defendido por ejemplo en el párrafo 635 de la Sentencia del 7 de mayo de 1977, en el asunto contra Tadić (IT-94-I-T) o en el párrafo 423 de la Sentencia del 22 de febrero de 200 I, en el asunto contra Kunarac y otros (IT-96-23 et IT-9623/I-A).

17 Definición que ya había aportado el TPIR y la misma CPI, el primero en el párrafo 580 en el asunto contra Akayesu. La Corte Penal en los párrafos 395-398 de la Decisión de la Sala preliminar I en el asunto Katanga (ICC-OI/04-OI/07-7I7-T). 
Soledad Torrecuadrada García-Lozano

Los avances de la jurisprudencia internacional contra la impunidad de las violaciones en los conflictos armados

to y no individualmente, con el propósito de "looking at their cumulative effect. Although individual acts may not be inhumane, their overall consequences must offend humanity in such a way that they may be termed 'inhumane ${ }^{\text {"'18. }}$. Debe analizarse, del mismo modo que en el genocidio, el conjunto de los comportamientos realizados, las violaciones y las agresiones sexuales, para averiguar si nos encontramos en presencia del crimen de lesa humanidad.

Los elementos que conforman la violación como constitutiva de crimen de lesa humanidad, según aprecia la CPI en la Sentencia Katanga, basándose en los elementos de los crímenes, son dos: uno objetivo y otro subjetivo. El primero es de nuevo la violación o agresión sexual. El segundo, el conocimiento de participar en una empresa criminal (artículo 7. I) g)-I del Estatuto de Roma), tal y como establece el artículo 30 del Estatuto de Roma ${ }^{19}$. El TPIY indicó lo que ha de entenderse por el conocimiento de los autores de su participación en una finalidad crimina $\left.\right|^{20}$. Así, Mlado Radic fue acu-

18 Vid. El párrafo 622 de la Sentencia de I4 de enero de 2000 , del TPIY, en el asunto contra Zoran Kupre[Ki], Mirjan Kupre[Ki], Vlatko Kupre[Ki], Drago Josipovi], Dragan Papi], Vladimir [Anti], también conocido como "Vlado" (IT-95-I6-T).

19 Vid. Los parágrafos 969-972 de la Sentencia de la Sala de Primera Instancia II de la Corte Penal Internacional, de 7 de marzo de 2014 en el asunto contra Germain Katanga, no ICC-OI/04-OI/O7.

20 Vid. Por ejemplo en el parágrafo 403 de la Sentencia de la Sala de Primera Instancia del Tribunal de Yugoslavia de 2 de noviem- sado de violación como crimen de lesa humanidad ${ }^{21}$, al considerar el Tribunal suficientemente probado que las detenidas en el campo de Omarska, del que era jefe de un equipo de guardias, fueron víctimas de violación (párrafo I82). Su responsabilidad derivaba del papel sustantivo que había desempeñado en ejercicio de su cargo en el campo en cuestión. Zoran Žigić, otro de los acusados por los hechos acaecidos en Omarska, alegaba un móvil personal - actuaba por un instinto heredado de su trabajo como policía antes de la guerra- (párrafo 462), para evitar la identificación de la participación en una empresa militar y, con ello, de la consideración del crimen de lesa humanidad.

La Sala de Apelaciones del TPIY desestimó la argumentación, al entender que el móvil particular que conduce al autor a perpetrar los comportamientos en cuestión, no es un elemento del crimen de lesa humanidad, a diferencia de la intención de perpetrar un ataque generalizado, que sí lo es. El móvil puede ser relevante en el momento de fijar la pena correspondiente, cuando se aprecian las circunstancias atenuantes y agravantes a estos efectos (párrafo 463).

bre de 200I, en el asunto contra Miroslav Kvočka, Mlađo Radić, Zoran Žigić y Dragoljub Prcać (IT-98-30/I-A).

21 Vid. La sentencia de la Sala de Apelaciones del Tribunal de Yugoslavia de 28 de febrero de 2005 en el asunto contra Miroslav Kvočka, Mlađo Radić, Zoran Žigić y Dragoljub Prcać (IT-98-30/I-A). 


\section{LA VIOLACIÓN COMO CRIMEN DE GUERRA}

La violación también se ha considerado un crimen de guerra, al tratarse de un comportamiento contrario a las leyes y usos de la guerra aludidos en las Convenciones de Ginebra y antes de ello en la Convención II de La Haya de I899, relativa a las Leyes y Usos de la Guerra Terrestre, cuyo Reglamento anexo precisamente contenía esas leyes y usos. A finales del siglo xx, se incorporaría expresamente la violación como una de las manifestaciones del crimen de guerra, lo que ha servido a la CPI para perfilar en su jurisprudencia los elementos de la violación para ser considerada de este modo.

En la sentencia contra Jean-Pierre Bemba Gombo ${ }^{22}$, la CPI afirma que la violación también puede ser calificada como crimen de guerra, atendiendo a sus elementos contextuales. Afirmación que no resulta novedosa si tenemos en cuenta que, a diferencia de lo visto en el epígrafe anterior, para considerar la presencia de un crimen de guerra, ha de cometerse en transcurso de conflicto armado internacional o interno y el violador ha de ser consciente de la presencia "de circunstancias de hecho que establecían la existencia de un conflicto armado"23.

22 Sentencia de 2 I de marzo de 2016 de la Sala de Primera Instancia III en la Situación en la República Sudafricana, en el asunto del Fiscal contra jean-Pierre Bemba Gombo (ICC-0I/05-0I/08).

23 El artículo 8.2.b) xxii)-I y artículo 8.2.e) vi)-I de los elementos de los crímenes del
En la sentencia en el caso Tadič, el TPIY se planteó que hay comportamientos que podrían ser constitutivos de crimen de guerra y de lesa humanidad. Sin embargo, el hecho de que se incorporasen las dos en el Estatuto de este Tribunal implica:

\section{"their intention that those war crimes which, in addition to targeting civil- ians as victims, present special fea- tures such as the fact of being part of a widespread or systematic practice, must be classified as crimes against bumanity and deserve to be punished accordingly" ${ }^{24}$.}

Afirmación que no resulta novedosa, porque el elemento contextual al que se refiere es la suma de aquellos que nos sirven para identificar la presencia de dicho conflicto, que en realidad son dos: la identificación de la situación conflictual y la organización por las autoridades gubernamentales o por grupos armados. En relación al primero, se utiliza la "definición Tadič" de conflicto, según la cual este existe siempre que se recurre al uso de la fuerza interestatal o interno ${ }^{25}$, lo que

Estatuto de Roma. Vid. En: https://wwwi. umn.edu/humanrts/

instree/S-iccelementsofcrime.html

24 Es el párrafo 286 de la sentencia de la Sala de Apelaciones del Tribunal de Yugoslavia del I5 de julio de 1999, en el asunto (IT-94I-A) contra Duŝko Tadič.

25 Párrafo 70 de la sentencia relativa a la apelación de la defensa sobre la excepción 
Soledad Torrecuadrada García-Lozano

Los avances de la jurisprudencia internacional contra la impunidad de las violaciones en los conflictos armados

nos conduce a la jurisprudencia de la CIJ, que en la sentencia en el caso de las actividades militares y paramilitares en y contra Nicaragua (fondo), contiene un verdadero manual sobre el principio que prohíbe la amenaza y el uso de la fuerza armada.

En el caso de la República Democrática del Congo, nos encontramos ante un conflicto armado internacionalizado, lo que no implica que todas las agresiones sexuales que se cometan se consideren, a estos efectos, como crímenes de guerra. La CPI en los asuntos Lubanga, Katanga y Bemba afirmó que un crimen de guerra requiere que los crímenes considerados sean atribuibles a grupos armados organizados ${ }^{26}$. El problema es su identificación, pues carecemos de un concepto jurídicamente determinado al respecto, en vista de lo cual, la Corte reitera sus palabras expresadas en los casos Lubanga y Katan- ga en las que exigía que estos grupos presentasen:

"un degré d'organisation suffisant pour leur permettre de mener un conflit armé prolongé et pour être capable de mettre en ouvre les dispositions du droit humanitaire applicables à ce type de conflit» ${ }^{27}$.

El grado de organización, por tanto, ha de ser flexible y, en cada caso concreto, debe estudiarse su presencia o ausencia. En conclusión, podremos hablar de crímenes de guerra cuando se produzcan violaciones en el transcurso de conflictos armados internos o internacionales, atribuibles a los grupos contendientes. prejudicial de incompetencia, en el asunto del Fiscal c. Dusko Tadič, alias "Dule" (Iт94-I), del 2 de octubre de 1995.

26 En este sentido, el Estatuto de Roma (artículo 8.2.f) requiere la existencia de un conflicto en el que se enfrenten grupos armados organizados.
27 Es el párrafo II85 de la Sentencia de la Corte Penal Internacional del 7 de marzo de 20I4, en el asunto contra Germain Katanga (ICCor/O4-0I/07). Original en francés. 


\section{LA IRRELEVANCIA DEL CONSENTIMIENTO DE LA VÍCTIMA}

Las agresiones sexuales se caracterizan en la mayoría de los sistemas jurídicos por ser un atentado a la libertad sexual de las personas, en que se emplea la violencia o la intimidación (así lo establece, por ejemplo, el artículo i78 del Código Penal español). Esto implica, necesariamente, la ausencia de un consentimiento libre, responsable y real al comportamiento sexual por parte de la víctima, pues parece razonable que en el transcurso de un conflicto armado, el temor es una constante entre la población civil, lo que, en este contexto, provocará el sometimiento de las víctimas que, razonablemente, accederán a las exigencias de su violador por miedo a males mayores para sí o para su familia. Conscientes de ello, en las Reglas de Procedimiento de la CPI, taxativamente se proclama la incapacidad de la víctima para consentir válidamente debido a la situación contextual por la que está atravesando, sin que pueda desprenderse de su silencio o de la falta de resistencia a una agresión de esta naturaleza.

Es evidente que ante la presencia del consentimiento se evitaría la aplicación del tipo de agresión sexual o violación y, en consecuencia, no se incurriría en genocidio, lesa humanidad o tortura, derivados de aquella, según lo reflejado en páginas anteriores. Sin embargo, los TPIs han seguido una lógica diferente en este punto, derivada del contexto conflictual en el que los hechos se producen. Así, han partido de la presunción de ausencia de consentimiento cuando la agresión sexual viene acompañada de los elementos antes vistos, que permiten su tipificación como un crimen de genocidio, lesa humanidad o torturas (Furundijza — TPIY - parágrafo I80 y Akayesu —TPIR- párrafo 597).

Por lo demás, sabido es por el Derecho Internacional general que el consentimiento no es una circunstancia que excluya la ilicitud cuando se trata de vulnerar una norma imperativa (artículo 26 del proyecto de artículos de la CDI sobre responsabilidad internacional de los Estados), que es precisamente el supuesto ante el que nos encontramos en el genocidio, de crímenes de guerra, la tortura o de lesa humanidad. Por lo que la aplicación de esta consideración nos conduce a idéntico resultado que la jurisprudencia internacional.

Además, la existencia o no de consentimiento es difícilmente demostrable ante un tribunal. No es, por ello, de extrañar que las reglas de procedimiento y prueba de los TPIs sigan la línea recién indicada. Así, el TPIY y TPIR incorporan unos principios sustantivamente idénticos en este punto (Regla №96), al proclamar que no se requiere prueba testifical de la violación por parte de otras personas distintas de la víctima (lo que procedería en aquellos supuestos en los que los hechos se hubieran producido públicamente. Por su 
Soledad Torrecuadrada García-Lozano

Los avances de la jurisprudencia internacional contra la impunidad de las violaciones en los conflictos armados

parte, la CPI incorporó las enseñanzas de los TPIY y TPIR, añadiendo de forma explícita "la dimensión de género en otros tipos constitutivos de crímenes de lesa humanidad" (OJINaga, 2002), lo que se advierte igualmente en sus reglas de procedimiento y prueba (Regla 70), que establecen los principios aplicables a la administración de justicia en los casos de violencia sexual.

Por su parte, la Sala de Apelaciones del TPIY, en el caso Kunarak, fue un paso más allá, al responder que la violencia o intimidación a la víctima no tiene por qué estar presente en el momento en el que se produce la violación, pudiendo resultar anterior a ella, pues:
"In this context, the Appeals Chamber further refers back to its finding that the coercive circumstances of this case made consent to the sexual acts by the Appellants impossible. This argument is, therefore, without merit and is rejected" 28 .

Queda así aclarada la irrelevancia del consentimiento en las agresiones sexuales que se producen en los conflictos armados, siempre que se reúna el resto de los requisitos establecidos para el tipo delictivo en el que tal comportamiento se encasille.

\section{Conclusiones}

Que los conflictos armados afectan de modo diferente, en función del género, es una evidencia contrastada. Tradicionalmente, las mujeres formaban parte de la población civil, y su violación se ha venido justificando como botín de guerra y, en tiempos más próximos, como una necesidad biológica de los contendientes. En los últimos decenios, la situación ha cambiado, pues si bien las mujeres siguen siendo en su mayoría parte de la población civil, también pueden participar activamente en las acciones bélicas, incluso dentro de los ejércitos estatales. Esto ha ampliado

28 Vid. Sentencia del I2 de junio de 2002, de la Sala de Apelaciones del Tribunal de su exposición a la violación, pues la práctica nos indica que ya no son solo víctimas de las filas enemigas, también de los propios compañeros o incluso de quienes integran las operaciones de mantenimiento de la paz. Por tanto, la vulnerabilidad frente a este tipo de crímenes no solo se mantiene, sino que se ha multiplicado, atendiendo a los posibles autores de estos comportamientos.

Uno de los aspectos olvidados en relación a las agresiones sexuales es el relativo a las menores de edad, al no incorporarse diferencia alguna en los

Yugoslavia, en el caso conocido como Foča, contra Kunarac, Kovac y Vukovic, p. I22, párrafo 409. 
tipos, atendiendo a la edad de las víctimas, cuando existe acuerdo acerca de que las consecuencias de uno de estos crímenes (tanto físicas como psicológicas) son diferentes en niñas y en mujeres adultas. A diferencia de lo que ocurre con el reclutamiento forzoso, tipificado como delito cuando afecta a menores de I5 años (en el Estatuto de Roma y en el Protocolo Facultativo de la Convención sobre los Derechos del Niño), en el caso de violación no se aprecia una diferencia en la regulación internacional de este delito cuando las víctimas son mayores o menores de edad. Los tribunales internacionales han conocido de la violación de mujeres y niñas, sin atribuir ningún agravante al último comportamiento respecto del primero ${ }^{29}$. Sin embargo, deberíamos introducir esta sensibilidad debido a la diferente afectación que tienen estas violaciones en función de la madurez de la víctima, así como la gravedad que el mismo crimen tiene desde cualquier perspectiva que se intente analizar, especialmente cuando observamos que en algunas contiendas se están produciendo violaciones a niñas de muy corta edad.

Las mujeres y niñas son las víctimas mayoritarias pero no exclusivas de las violaciones. Pese a ello, con independencia del género de las víctimas, en los conflictos armados nos encontra-

29 El artículo I83 del Código Penal español contempla penas especiales cuando se producen abusos sexuales respecto de menores de 16 años. mos ante una autoría siempre masculina. Cuando las víctimas son varones adultos, se realiza con el propósito de producir la mayor de las humillaciones.

La violencia sexual tiene consecuencias físicas y psicológicas, pudiendo las consecuencias de aquella evidenciarse en ambos planos. En ocasiones, como las de la antigua Yugoslavia, con los embarazos forzados; en otras, como el caso de las "mujeres de solaz", con la pérdida de su capacidad reproductora debido a las numerosas violaciones prolongadas en el tiempo.

En lo que se refiere a los daños psicológicos, suelen convivir con traumas prolongados en el tiempo, fobias, autolesiones e insomnio, entre otros. A todo ello ha de unirse la estigmatización en las comunidades de origen, que si bien ven a los caídos, heridos o mutilados en la contienda, como héroes, quienes han sido víctimas de abusos sexuales reciben el tratamiento contrario, pese a ser igualmente víctimas de la guerra.

Al contrario de otras víctimas de los conflictos armados, las víctimas de violaciones, lejos de recibir reconocimiento alguno, han tenido que asumir el silencio o la marginación en sus comunidades de origen, además de ser víctimas de enfermedades como el vir o de otras producidas como consecuencia de las violaciones reiteradas. El daño psicológico derivado del horror del que han sido víctimas no tiene reparación posible, pero son las últimas en recibir tratamiento para poder sobrellevar su 
Soledad Torrecuadrada García-Lozano

Los avances de la jurisprudencia internacional contra la impunidad de las violaciones en los conflictos armados

situación del mejor modo posible y eso cuando existe esta posibilidad.

En todo caso, a pesar de los problemas que la situación actual presenta, en los últimos años hemos avanzado mucho, pues por primera vez se ha juzgado la responsabilidad criminal individual por delitos sexuales. Se ha definido el concepto de violación, incluso hemos sido testigos de conceptualizaciones diferentes a este respecto entre el TPIR y TPIY, y hemos podido progresar por este camino.

Lo anterior evidencia la ineficacia de las normas que prohíben la violencia sexual en los conflictos armados, afirmación que no resulta novedosa, pues fue puesta de relieve hace casi veinte años por la Relatora Especial sobre la Violencia contra las Mujeres, Causas y Consecuencias, en su informe del 26 de enero de $1998^{30}$. Deberíamos, en este punto, comprometernos para dotar a estas proscripciones de cuantas medidas fueran necesarias para procurar su eficacia, porque solo de este modo podremos intentar prevenir estos crímenes, cuya reparación resulta tan problemática. Está visto que las llamadas del Consejo de Seguridad a las partes en conflicto, para que pongan fin a estos comportamientos, no

30 UN, Doc E/CN.4/1998/54, que puede verse en https://documents-dds-ny.un.org/doc/ UNDOC/GEN/G98/I02/94/PDF/G9810294. pdf?OpenElement , parágrafos 95 y ss., páginas $24 \mathrm{y}$ ss. obtienen resultados, por lo que debemos pensar en otro tipo de medidas que puedan resultar eficaces en el camino de la prevención.

Un problema sumado al anterior y más difícil de atajar deriva de la utilidad de las violaciones para conseguir los fines pretendidos por los agresores, que afectan psicológicamente al enemigo, en mayor medida, que el logro de un objetivo militar. Además, se trata de comportamientos muy baratos, pues a diferencia de otros no precisan de armamento alguno, por lo que si añadimos esto a lo anterior, no podemos ser muy optimistas en que la situación se altere y logremos controlar y reducir su aplicación en conflictos actuales o futuros. Se ha comprobado que la violación es un arma de guerra muy barata y con una inmensa eficacia.

Los avances que precisamos son muchos y las fuerzas escasas, máxime teniendo en cuenta la cantidad de frentes que tenemos abiertos para lograr un mundo mejor, pero quizás a este deberíamos dotarle de preferencia porque los daños que produce, no solo en las víctimas directas, son elevadísimos. Pensemos, por ejemplo, en el futuro que espera a los niños nacidos de embarazos forzados en los casos de Ruanda o Bosnia, estos son otras víctimas que merecen un estudio monográfico, pero que no podemos dejar de plantear en este punto. 


\section{BibLIOGRAFÍA}

AA.VV. (1920). "Commission on the responsibility of the authors of the war and on enforcement of penalties. Report presented to the preliminary peace conference march 29, 1919”. American Journal of International Law, I4, pp. 95 y ss.

Abril Stoffels, R. (20I2). "Las resoluciones del Consejo de Seguridad sobre mujer, paz y seguridad: objetivos, logros y desafíos para las operaciones de paz”, en Jorge Cardona Llorens y otros. Estudios de derecho internacional y de derecho europeo en homenaje al profesor Manuel Pérez González. Valencia: Tirant Lo Blanch, tomo I, p. 6r y ss. Akman, W. (2013). "Atrocidades contra la humanidad durante la guerra de liberación en Bangladesh: un caso de genocidio". Revista de Estudios sobre Genocidio, № 4, pp. 50 y ss.

Амвоs, K. (20I2). "Violencia sexual en conflictos armados y derecho penal internacional". Cuadernos de política criminal, número I07, II, Segunda época, pp. 5 y ss.

Anónimo (2005). Una mujer en Berlín. Barcelona: Anagrama.

Aydelott, D. (1993). "Mass rape during war: prosecuting bosnian rapists under International Law". Emory International Law Review, vol. 7, pp. 585 y ss.

Barrow, A. (20I0). "UN Security Council Resolutions 1325 and I820: constructing gender in armed conflict and international humanitarian Law". International Review of the Red Cross, vol. 92, № 877 , p. 22I y ss.,
Bassioini, M. Сh. y McCormick, M. (1996). "Sexual Violence. An Invisible Weapon of War in the Former Yugoslavia”. Occasional Paper №I, International Human Rights Law Institute. DePaul University College of Law.

Bou Franch, V. (20I5), "El crimen internacional de esclavitud sexual y la práctica de los 'matrimonios forzados". Anuario Español de Derecho Internacional, vol. 3I, pp. 65 y ss.

Bou Franch, V. (20I2) "Los crímenes sexuales en la jurisprudencia internacional”. Revista Electrónica de Estudios Internacionales, no 24, p. I y ss.

Bustamante Arango, D. M. (20I4). "La violencia sexual como tortura. Estudio jurisprudencial en la Corte Interamericana de Derechos Humanos". Revista de la Facultad de Derecho y Ciencias políticas, vol. 44, no I2I, pp. 46I y ss.

Chinkin, CH. (200I), "Editorial Comments. Women's International Tribunal on Japanese Military Sexual Slavery”. American Journal of International Law, vol. 95, pp. 335 y ss.

Chinkin, Ch. (1994). "Rape and sexual abuse of women in International Law". European Journal of International Law, no 5 , pp. I y ss.

McGlyn, C. (2008). "Rape as 'Torture' Catherine MacKinnon and Questions of Feminist Strategy". Feminist Legal Studies, noI6, 2008, pp. 7r y ss.

De Beauvoir, S. (1949). Le deuxième sexe. París: Gallimard.

de Vito, D.; Gill, A. y Short, D. (2009). "El delito de violación tipificado como 
Soledad Torrecuadrada García-Lozano

Los avances de la jurisprudencia internacional contra la impunidad de las violaciones en los conflictos armados

genocidio". Sur, Revista internacional de los derechos humanos, № IO, pp. 29 y ss. DíAs, L.A. (20I4). "Violencia sexual contra niños y niñas menores de quince años en el caso Lubanga: análisis crítico y una propuesta de solución”. Anuario Iberoamericano de Derecho Internacional Penal, vol. 2, p. 103 y ss.

FIORI, M. (2007). "Les 'camps de viol de Foča': La jurisprudence du TPIY sur une page sombre de la guerre”. Hague Justice Journal|Journal Judiciaire de La Haye, vol. 2, no3.

Frieyro de Lara, B. (20I2). "Mujeres: objetivo militar", en M. Robles Carrillo, Género, conflictos armados y seguridad. La asesoría de género en operaciones. Universidad de Granada y Mando de Adiestramiento y Doctrina, pp. 63 y ss.

Frulli, M. (2008). "The Special Court for Sierra Leone Recognizes Forced Marriage as a 'New' Crime against Humanity”. Journal of International Criminal Justice, 6, pp. IоI3 y ss.

Gómez IsA, F. (2000). La participación de los niños en los conflictos armados. El Protocolo Facultativo a la Convención sobre los Derechos del Niño. Bilbao: Cuadernos de Deusto.

Herrmann, I., y Palmieri, D. (2010). "Between Amazons and Sabines: a historical approach to women and war'. International Review of the Red Cross, vol. 92, no 877, pp. 19 y ss.

Kucukalic Ibrahimovic, E. (20I4). "Las mujeres violadas en la Guerra de Bosnia, dobles víctimas del conflicto veinte años después". Instituto de Estudios Estratégicos, 35/20I4. Recuperado de: http:// www.ieee.es/Galerias/fichero/docs_opi-
nion/20I4/DIEEEO35-20I4_GuerraBosnia_DDHH_EsmaKucukalic.pdf

Magallón Portolés, C. (2008-2009). "Mujer, Paz y Seguridad: un balance de la Resolución I325”. Anuario CEIPAZ, no 2, pp. 63 y ss.

Mayumi Yasunaga, M.E. (2016). "Las mujeres de confort: un acuerdo histórico". Instituto Español de Estudios Estratégicos. Recuperado de: http://www.ieee. es/Galerias/fichero/docs_opinion/2016/ DIEEEOII-20I6_Mujeres_Confort_ MMayumi.pdf

McGlynn, C. (2009). "Rape, torture and European Convention on Human Rights”. International \& Comparative Law Quarterly, vol. 58-3, pp. 565 y ss.

McGlynn, C. (2008). "Rape as 'Torture' Catherine MacKinnon and Questions of Feminist Strategy". Feminist Legal Studies, noI6, pp. 7I y ss.

Noulo, M. (2009). "The United Nations Responses to the Sexual Abuse and Exploitation of Women and Girls by Peacekeepers during Peacekeeping Missions". Berkeley Journal of International Law, vol. 27-I, pp. 126 y ss.

NúÑez Marín, R.F., y Zuloaga Jaramillo, L.N. (20II). "La violencia sexual como una forma de tortura en el derecho internacional de los Derechos Humanos". Criterio Jurídico, vol. II, no I, pp. 135 y ss. OJinaga Ruiz, Ma.R. (2002). "La prohibición y criminalización en Derecho Internacional de las violencias sexuales contra mujeres civiles en conflictos armados". Boletín de la Facultad de Derecho de la UNED, no 19 , pp. 199 y ss.

Omanyondo Ohambe, M.C.; Berckmans Bahananga Muhigwa, J. y Mulyum- 
ва Wa Mамва, B. (2005). Women's Bodies as a Battleground: Sexual Violence Against Women and Girls During the War in the Democratic Republic of Congo South Kivu (1996-2003), ed. Réseau des Femmes pour la Défense des Droits et la Paix International Alert. Rodríguez, O. (199I). Etnias, Imperios y Antropología. Caracas: Universidad Central de Venezuela.

Rose, C. (2009). "Troubled Indictments at the Special Court for Sierra Leone The Pleading of Joint Criminal Enterprise and Sex-based Crimes". Journal of International Criminal Justice, 7, pp. 353 y ss.

SAкамото, R. (200I). “The Women's International War Crimes Tribunal on Japan's Military Sexual Slavery: A Legal and Feminist Approach to the 'comfort Women' Issue”. New Zealand Journal of Asian Studies, vol. 3-I, pp. 49 y ss;

SÁnchez, G. (2010). Amazonia y sus etnias. Create Space.

Satya Kalra, M. (200I). "Forced Marriage: Rwanda's Secret Revealed". Journal of International Law and Policy, pp. 197 y ss.

Sivakumaran, S. (2010). "War Crimes before the Special Court for Sierra Leone Child Soldiers, Hostages, Peacekeepers and Collective Punishments". International of International Criminal Justice, 8, pp. 1009 y ss.

Slater, R. (20I2). "Gender Violence or Violence against Women? The Treatment of Forced Marriage in the Special Court of Sierra Leone”. Melbourne Journal of International Law, I3, pp. 732 y ss.

Solana, M. (2015). "Mujeres, paz y seguridad: lejos de las aspiraciones de la Resolución I325”. Documentos del Real Instituto Elcano, ARI/44/2015, I5 de septiembre.

Tachou-Sipowo, A.G. (20I0). “The Security Council on women in war: between peacebuilding and humanitarian protection". International Review of the Red Cross, vol. 92, no 877, p. 197 y ss.

Toy-Cronin, B.A. (20I0). "What is forced marriage? Towards a definition of forced marriage as a crime against humanity". Columbia Journal of Gender and Law, no I9, pp. 539 y ss.

Turshen, M. y Twagiramariya, C. (1998). What Women Do in Wartime. Zed Press, pp. 73 y ss.

Vanyó Vicedo, R. (2016). El horizonte 1325 en Derecho Internacional: cartografía del posconflicto con perspectiva de género. Pamplona: Aranzadi. 